\title{
Concepciones culturales sobre la salud mental de personas que asisten a procesos terapéuticos psicológicos
}

\author{
Cultural conceptions about mental health of \\ people on psychological therapeutic process
}

\author{
Teresa Margarita Torres-López ${ }^{1}$, Jazmín Aranzazú Munguía Cortés², \\ Carolina Aranda Beltrán ${ }^{3}$, José Guadalupe Salazar Estrada ${ }^{4}$ \\ Universidad de Guadalajara, México
}

\begin{abstract}
Resumen. El objetivo fue analizar las concepciones culturales sobre la salud mental de personas adultas que asisten a procesos terapéuticos psicológicos de Guadalajara, México. Método: Participaron 77 personas seleccionadas por muestreo propositivo. Se aplicaron las técnicas de listados libres y pile sort. El análisis fue de consenso, conglomerados jerárquicos y escalas multidimensionales. Resultados: Para los participantes la salud mental es el bienestar, la salud y la cordura que se logra con el apoyo de profesionales e instituciones de salud. Se conceptualizó además como un estado de equilibrio, tranquilidad, paz, armonía, amor, felicidad, plenitud, entusiasmo, seguridad y congruencia. Por el contrario, la falta de salud mental puede ocasionar algunos trastornos mentales (neurosis, psicosis, depresión). Se observaron diferencias por género y un mayor consenso en mujeres que en hombres, lo que permite proponer bases diferenciales en la implementación de programas de promoción de la salud mental.
\end{abstract}

Palabras clave. Salud mental, procesos terapéuticos, concepciones culturales, promoción de la salud mental, psicología de la salud.

Abstract. The objective was to analyze mental health cultural conceptions' of adult people with experiences on psychological therapeutic process from Guadalajara, Mexico. Method: 77 persons selected by purposive sampling were interviewed. It was applied free listing and pile sort technics. The analysis was by consensus, hierarchical cluster and multidimensional scaling. The participants' conceptualized mental health like the well-being, health and sanity obtained by the professional and institutions health support. For them, mental health is the balance, tranquility, pace, harmony, love, happiness, plenitude, enthusiasm, confidence and agreement. In other hand, lack of mental health would be generating some mental illness (neurosis, psychosis, depression). The results showed genre differences and high woman consensus than the men, that allowed suggest differential basis for design promoting programs of mental health.

Keywords. Mental health, therapeutic process, cultural conceptions, promoting mental health, health psychology.

\footnotetext{
${ }^{1}$ Teresa Margarita Torres López. Departamento de Salud Pública del Centro Universitario de Ciencias de la Salud de la Universidad de Guadalajara. Direccion postal: Sierra Mojada 750, puerta 1, Colonia Independencia. CP. 44340, Guadalajara, Jalisco, México. E-mail: tere.torres.cucs@gmail.com 2 Jazmín Aranzazú Munguía Cortés. Departamento de Psicología Aplicada del Centro Universitario de Ciencias de la Salud de la Universidad de Guadalajara. E-mail: jazminmunguia@gmail.com

${ }^{3}$ Carolina Aranda Beltrán. Departamento de Salud Pública del Centro Universitario de Ciencias de la Salud de la Universidad de Guadalajara. E-mail: caranda2000@yahoo.com.mx

${ }^{4}$ José Guadalupe Salazar Estrada. Departamento de Salud Pública del Centro Universitario de Ciencias de la Salud de la Universidad de Guadalajara.. E-mail: jsalazar43@gmail.com
}

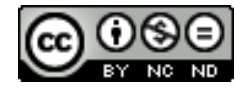

Esta obra está bajo una licencia de Creative Commons Reconocimiento-NoComercial-SinObraDerivada 4.0 Internacional. 


\section{Introducción}

Las diversas causas de los problemas de salud mental y de los trastornos mentales incluyen no sólo características individuales (tales como la capacidad para gestionar nuestros pensamientos, emociones, comportamientos e interacciones con los demás), sino también factores sociales, culturales, económicos, políticos y ambientales; las políticas nacionales, la protección social, el nivel de vida, las condiciones laborales y los apoyos sociales de la comunidad (Organización Mundial de la Salud [OMS], 2013).

Los programas de promoción y atención de salud mental existentes por parte del sector salud de México han tenido un impacto limitado. La mayoría de los programas implementados se orientan hacia la atención de problemas específicos de enfermedad mental, dejando de lado la promoción y fomento de la misma. Además, no se toma en cuenta la perspectiva de los sujetos a los cuales se dirigen los programas, es decir, los actores implicados.

La promoción de la salud mental procura fomentarla mediante el mejoramiento de los recursos, habilidades y fortalezas de las personas, familias, comunidades e instituciones. También, busca fomentar el establecimiento de condiciones de vida adecuadas, así como "empoderar" a los actores sociales para que esas condiciones puedan lograrse (OMS, 2005). La Organización Mundial para la Salud (OMS) define a la salud mental como un estado de bienestar en el que la persona materializa sus capacidades y es capaz de hacer frente al estrés normal de la vida, de trabajar de forma productiva y de contribuir al desarrollo de su comunidad (OMS, 2004).

Los estudios que se han enfocado a identificar la conceptualización de la salud no sólo desde la visión de los expertos, sino de la población en general, destacan un creciente interés por la perspectiva de las personas que no cuenta con formación académica en el campo de la salud, es decir, de los profanos o legos. La correcta promoción de la salud metal, considera que las estrategias e intervenciones terapéuticas, profilácticas y de promoción, tienen que basarse en pruebas científicos y en prácticas óptimas, teniendo en cuenta consideraciones de carácter cultural (OMS, 2013).
Un enfoque teórico y metodológico que permite el acercamiento a las conceptualizaciones culturales de la población es el análisis de consenso cultural. El cual estudia cómo las personas de diferentes culturas adquieren información sobre el mundo, cómo la procesan, toman decisiones y actúan en formas consideradas apropiadas para otros miembros de su cultura (transmisión cultural).

La cultura es un conjunto de conocimientos, creencias y conductas compartidas que constituyen los elementos normativos de un grupo. La teoría del consenso es un conjunto de técnicas analíticas y modelos que pueden usarse para estimar los saberes culturales. El conocimiento individual, o competencia cultural individual, puede ser analizado desde el acuerdo entre los integrantes de un grupo determinado. Se parte de la idea de que las personas son culturalmente competentes en un área específica de un objeto social en la que son expertos no sólo por su conocimiento científico, sino también, y sobre todo, por los saberes culturalmente compartidos El análisis de consenso es teoría y método, ya que como teoría especifica las condiciones bajo las cuales un mayor acuerdo entre los individuos, indica un mayor conocimiento sobre un dominio cultural. Y como método permite evaluar el grado del conocimiento de los informantes (Weller, 2007).

Los dominios culturales son categorías significativas de conocimiento para una cultura en particular. Son creados por la población, a partir de reglas cognitivas que permiten organizar las ideas, cosas y formas que tienen en mente, además de crear modelos para percibir, relacionar e interpretar la información (Bernard, 2006).

Se accede a ellos con el uso de técnicas estructuradas de obtención de información (listados libres, card sorting, formación de triadas o pares, entre otras). Lo que permite generar información cuantificable, con el fin de comprobar los tres supuestos del la teoría del consenso cultural: a) verdad común: se asume que todos los informantes provienen de una cultura común. b) independencia local: supone que las respuestas de cada informante son independientes de las respuestas de los otros informantes. c) la homogeneidad en los items: cada informante tiene una competencia cultural fija sobre cada término (Weller, 2007). 
En el campo de la salud se han realizado diversos estudios con este enfoque, las temáticas abordadas son sobre dengue (Torres-López, Guerrero \& Salazar, 2012; Torres-López, Soltero-Avelar \& Herrera, 2012), influenza humana (Torres-López, Matsui \& Aranda, 2012) y Virus de Inmunodeficiencia Humana (VIH) (Torres-López \& Munguía, 2012).

En un estudio en torno a la conceptualización de la salud y enfermedad de personas adultas de la ciudad de Guadalajara, México, se encontró en el concepto de salud una visión holística que incluye aspectos físicos, emocionales y espirituales. La limpieza y el desarrollar un trabajo son esenciales en la prevención de la enfermedad. Los varones destacaron la importancia de no tener vicios, mientras que las mujeres resaltaron el tener buenas relaciones interpersonales. En el concepto de enfermedad hay un enfoque biológico y social que incluyó la deficiencia física, los estados emocionales alterados, así como el tratamiento y la atención de la enfermedad. Los varones señalaron los gastos económicos que implica su atención, mientras que las mujeres el agotamiento que conlleva el trabajo de atención de los enfermos (Torres-López, Munguía, Pozos \& Aguilera, 2010).

En cuanto a estudios sobre la construcción de significados acerca de la salud mental, se reporta uno en población adulta de una comunidad urbana marginal de la ciudad de México (Mora, Flores, De Alba \& Marroquín, 2003). La población participante señaló aspectos emocionales placenteros, relacionándolos con estar sano (tranquilidad, confianza, respeto, descanso, alivio, cariño y comprensión) y por el contrario los negativos para referirse a la enfermedad mental (sufrimiento, locura, depresión, sentirse mal).

Otro estudio analiza las percepciones de la población adulta mexicana acerca de sus padecimientos. En ellas hay una visión global que abarca toda la esfera vivencial del individuo (aspectos físicos y emocionales). A la salud mental se le asocia a la capacidad de ser feliz, al optimismo, la libertad, voluntad y al entusiasmo de vivir. Además de mantener una relación armónica entre el individuo con la sociedad y con Dios. A la enfermedad mental se le asocia con estar loco, la locura comprende una conducta trasgresora de las normas de interacción social, la violencia, agresiones, no razonar $\mathrm{y}$ tener mal comportamiento (como fumar, decir groserías, incoherencia y en general expresarse mal). Lo que lleva finalmente a enfermar la mente (Berenzon \& Mora, 2005).

En un estudio realizado con población de Estocolmo, Suecia, se encontró que una buena salud mental incluye al bienestar, al empoderamiento, tener un buen estilo y proyecto de vida, balance entre el tiempo de trabajo y descanso. Los problemas de salud mental surgen cuando hay pensamientos negativos, desequilibrio entre el tiempo de trabajo y descanso, estrés, ansiedad, problemas en la infancia, enfermedades y características personales negativas (Åsbring, 2012).

Conocer las concepciones culturales sobre la salud mental que tiene la población permitirá fundamentar con bases más sólidas, los programas de promoción de la misma. El estudio incluyó a personas que han tenido la experiencia haber recibido terapia psicológica, considerando que este tipo de población se encuentra más sensibilizada a la utilidad de este tipo de servicios de atención a la salud mental. El objetivo es analizar las concepciones culturales sobre la salud mental de personas que asisten a procesos terapéuticos psicológicos de la ciudad de Guadalajara, México.

\section{Metodología}

Se realizó un estudio de análisis de consenso cultural; la codificación de la información en este enfoque implica un tratamiento estadístico a fin de obtener el análisis de consenso, lo anterior es un análisis cuantitativo de datos cualitativos, implica convertir palabras o imágenes en números (Bernard, 2006).

\section{Participantes}

El estudio se realizó en la ciudad de Guadalajara, México; los participantes fueron hombres y mujeres habitantes de dicha población, específicamente de una zona de clase media. Se integró una muestra de tipo propositivo (Suri, 2011). Los criterios para su selección fueron: mayores de edad, habitantes de Guadalajara, inclusión de ambos sexos, que hubieran recibido atención psicológica (de 
cualquier orientación) en un periodo de por lo menos tres meses. Fueron abordados en la vía pública ya que se pretendía obtener la visión de la población abierta.

Antes de cada entrevista se explicaron los objetivos del estudio y se obtuvo el consentimiento informado verbal, garantizándose a los informantes la confidencialidad y anonimato en la recolección y el análisis de la información. La participación de los entrevistados y entrevistadas se sujetó a su participación voluntaria.

El tamaño de la muestra se basó en el supuesto del modelo de consenso cultural para el estudio de patrones culturales. El cual plantea que en estudios de descripción cultural el tamaño de muestra no debe ser grande, debido a que la correlación promedio entre informantes tiende a ser alta (.5 ó más). Los dos factores que determinan el número de informantes son la competencia cultural esperada (concordancia entre las respuestas individuales y el patrón estimado de respuestas correctas del grupo) y el nivel de confianza aceptable para determinar la respuesta a la pregunta. Es necesario un mínimo de 17 informantes para clasificar el $95 \%$ de las preguntas correctamente, bajo un promedio de competencia cultural esperada de $.5 \mathrm{y}$ un nivel de confianza de .95 (Weller, 2007).

Los participantes fueron 77 personas en total, 40 mujeres y 37 hombres, sus edades fluctuaron entre 20 y 64 años, con $M=35.7$ años en las mujeres y $M=33.7$ años los hombres. El mayor número de personas (85\%) se ubicó en el grupo de edad de 20 a 50 años. Su ocupaciones fueron estudiantes, empleados y profesionistas (ninguno de ellos había estudiado o laboraba en áreas relacionadas con el sector salud). Todos reportaron haber acudido a terapia psicológica en periodos de tres meses a nueve años y medio, el 68\% de ellos asistió en un tiempo de tres a seis meses. Dos terceras partes dijeron conocer la existencia de problemas de salud mental tales como depresión, trastorno de déficit de atención, esquizofrenia, bipolaridad e intento de suicidio.

El proyecto de investigación que fundamentó el presente estudio fue evaluado y en su momento aprobado por el comité de ética e investigación del Instituto Regional de Investigación en Salud Pública del Departamento de Salud Pública de la Universidad de Guadalajara (registro IISO/CI/27/09).

\section{Instrumentos}

Las técnicas de recolección de datos fueron entrevistas estructuradas: una la técnica de listados libres y otra la técnica de pile sorting (Bernard, 2006). Ambas favorecen la expresión espontánea de los sujetos y por ello se espera que el contenido evocado esté más libre de racionalizaciones, sesgos de defensa o deseabilidad social (Ruiz, Ponce de León \& Herrera, 2001).

Los listados libres consisten en pedir a los informantes términos relacionados con una esfera conceptual particular: "Dime cinco palabras o frases que se te vienen la mente cuándo escuchas: salud mental", una vez escritas en el instrumento las palabras, se les pide una pequeña explicación de por qué dijeron cada una de ellas (las citas textuales usadas en el apartado de resultados provienen de estas explicaciones). Luego de obtenidas las listas de palabras se tabularon las frecuencias, lo que permitió escoger 20 términos de cada listado (el de hombres y el de mujeres), a fin de generar el instrumento pile sorting.

El pile sorting consiste en elaborar tarjetas para cada término y pedir a los informantes que formen grupos de tarjetas que contengan los términos más parecidos, sin importar el número de grupos generados. Después se les pide que etiqueten a cada montón con una palabra (o frase) que identifique el conjunto de las tarjetas seleccionadas.

El tiempo de aplicación en la primera fase fue de máximo cinco minutos, la segunda de 10 a 15 minutos, lo que facilitó la participación de las personas.

\section{Estrategia de análisis}

Las palabras obtenidas en los listados libres que se mencionaron más de una vez, fueron clasificadas con base a tres categorías: los elementos descriptivos (incluyó sinónimos y términos asociados), las prácticas (referido a acciones o funciones) y los atributos (tales como adjetivos o cualidades) (Ruiz et al., 2001). Un segundo análisis implicó, como ya se indicó, la sumatoria de frecuencia de las palabras a fin de generar el pile sorting, con las 20 palabras con mayor mención.

Los datos obtenidos en pile sorting fueron tabulados en matrices de palabra por palabra y sus valores fueron transformados a proporciones de similaridades. Las 
matrices resultantes fueron analizadas por separado para los valores agregados $\left(\right.$ AGPROX $^{5}$ ) y los valores individuales (INDPROX $\left.{ }^{6}\right)$.

En el caso de los valores agregados, las matrices fueron analizadas mediante conglomerados jerárquicos ${ }^{7}$; este método asigna palabras a dimensiones conceptuales de acuerdo a la similaridad percibida por los informantes. El grado de similaridad entre palabras se expresó en una gráfica de diagrama de árbol con niveles de correlación. Por convención, se definieron los siguientes niveles de correlación entre las palabras: fuerte entre 1 y .75; moderado entre .74 y .50; débil entre .49 y .25; y mínimo entre .25 y 0 .

En forma complementaria, se hizo la representación espacial en dos dimensiones de las similaridades de las palabras representadas en los árboles de conglomerados, mediante escalas multidimensionales no métricas. Con esta técnica se calculó el valor de stress como medida de verosimilitud de la representación de los conglomerados. Esta medida se define como la suma de correspondencias entre pares de objetos dentro de la representación espacial. En el trabajo se siguió la propuesta de Sturrock \& Rocha (2000) quienes sugieren para una representación de 20 términos un valor de corte de stress menor a 28 y para 15 términos un valor menor de .23 .

En el caso de los valores individuales, las matrices fueron analizadas mediante un modelo de consenso cultural ajustado a respuestas ordinales (Weller \& Mann, 1997). Este modelo estima la verosimilitud de que un sistema homogéneo de conocimientos predomine en una cultura, a partir de la correspondencia entre los conocimientos del grupo y los del individuo. Para el efecto, se corrieron análisis factoriales de componentes principales que agrupan a los individuos en función de sus respuestas. El criterio de verosimilitud para la obtención del consenso en los

\footnotetext{
${ }^{5}$ Contiene el promedio de las matrices de proximidad de todos los individuos.

${ }^{6}$ Contiene las matrices de proximidad de ítem por ítem correspondientes para cada participante.

${ }^{7}$ Los conglomerados jerárquicos son un conjunto de procedimientos para organizar objetos (o conceptos) dentro de una secuencia de particiones a partir de una base de datos visualizados por la proximidad (o distancia) entre dichos objetos. En este caso se realizó el análisis con base en el algoritmo de Johnson (1967 citado por Marseline \& Premalatha, 2013) con el método complete-linkage clustering, para un tipo de datos de similaridad.
}

modelos implica que el primer factor alcance una razón tres veces mayor a la del segundo factor, lo cual significa que hay una alta concordancia en las respuestas de los informantes.

Los distintos análisis se procesaron con el programa computarizado Anthropac v. 4.98.

\section{Confiabilidady validez de la información}

$\mathrm{Al}$ ser un estudio que implicó análisis mixto (de tipo cualitativo y cuantitativo) se consideraron dos tipos de control de calidad de la información. El primero es el de triangulación entendido como la combinación de aproximaciones en el estudio de los mismos fenómenos. Estas puede ser de tiempo (obteniendo información en diferentes momentos), espacio (en lugares diferentes), métodos, contrastación teórica y de investigadores (Flick, 2014). Esta última fue la que se aplicó, ya que en el proceso de análisis participaron todos los investigadores en forma separada, los que llegaron a los mismos resultados.

El análisis de consenso cultural se considera que la información es más confiable si genera resultados similares aún cuando proviene de múltiples informantes (ya que refleja que éstos poseen alta competencia cultural) (Weller, 2007).

\section{Resultados}

\section{Contenido de la concepción cultural de salud mental}

Las palabras más frecuentes sobre salud mental obtenidas en los listados libres se presenta en la Tabla 1.

Las mujeres citaron una mayor variedad de términos que los hombres. Las palabras ubicadas como sinónimos fueron las mismas para los dos géneros (bienestar, salud, cordura, un hombre comentó: "si estas bien mentalmente vas a hacer cosas correctas, no vas a tener consecuencias malas" términos descriptivos asociados a salud mental tiene que ver con profesionales de la salud (psicólogos y psiquiatras, una mujer citó: "él ayuda emocionalmente y orienta lo mejor que se pueda"), instituciones y procesos (terapia, diagnóstico, tratamiento, una mujer dijo al respecto: "vamos a estar tranquilos por los consejos que nos da el psicólogo" y otra apuntó: "para tener salud mentar debes de asistir a

\footnotetext{
${ }^{8}$ Las citas textuales de los informantes provienen de las explicaciones proporcionadas para cada término, en el instrumento de listados libres.
} 
Tabla 1

Palabras más frecuentes sobre salud mental obtenidas en los listados libres

\begin{tabular}{|c|c|c|}
\hline Términos & Mujeres & Hombres \\
\hline \multirow{9}{*}{$\begin{array}{l}\text { Descriptivos } \\
\text { - sinónimos } \\
\text { - asociados }\end{array}$} & bienestar / salud / cuerdo & bienestar / salud/ cordura \\
\hline & enfermedad mental / loco & \\
\hline & persona / cabeza / cuerpo & \\
\hline & psicología / terapia & psiquiatra / psicólogo / terapia / \\
\hline & doctores / diagnóstico & inteligencia / neurosis / psicosis \\
\hline & medicamento / internar & \\
\hline & mente / carácter / depresión & \\
\hline & comunidad / sociedad / escuela & \\
\hline & casa / familia & \\
\hline \multirow{14}{*}{ Prácticas y funciones } & apoyo / ayuda / orientación & \\
\hline & compartir / comprensión & capacitación / ejercicio físico \\
\hline & sincera / respeto / no egoísta & ser positivo \\
\hline & mejorar relaciones familiares & \\
\hline & serenidad / tranquilidad / armonía & tranquilidad / estabilidad \\
\hline & / paz / sentirse bien / estabilidad / & paciencia / paz / relajación \\
\hline & paciencia / agradable/ equilibrio & confianza / equilibrio \\
\hline & amor / ternura & felicidad / entusiasmo/ alegría \\
\hline & felicidad / ánimo / coraje & amor / plenitud \\
\hline & seguridad / libertad & conciencia / perdón \\
\hline & independencia/ decisión / congruencia & proyectos / realización / sabiduría \\
\hline & tristeza/ no está bien & tristeza / emoción \\
\hline & desequilibrado / incongruente & celos / egoísmo \\
\hline & emoción / enojo / malestar & preocupación / inquietud \\
\hline \multirow{2}{*}{ Atributos } & Éxito & es muy importante \\
\hline & Necesidad & es una ventaja \\
\hline
\end{tabular}

terapias de superación personal, familiares, personales y de valoración"), de atención a la salud y a la enfermedad mental, así como algunos trastornos mentales (neurosis, psicosis, depresión). Sólo las mujeres mencionaron aspectos sociales o comunitarios asociados a la salud mental (familia, comunidad, escuela) sobre lo cual una mujer mencionó: "interactuamos unos con otros, y si alguien está enfermo afecta a los demás".

En prácticas, fueron señaladas por las mujeres: la búsqueda de apoyo, ayuda y orientación ("es bueno recibir apoyo de alguien profesional, para que te puedan ayudar con tus problemas"), a fin de compartir ("el hacerlo nos da satisfacción y el servir a los demás nos hará felices"), buscar respeto y comprensión en forma no egoísta ("uno ya ve lo que está mal y empieza a comprender más a sus semejantes"), además de mejorar las relaciones familiares. Los hombres incluyeron la capacitación, el desarrollo de ejercicio físico y el ser positivo ("al pensar positivamente, las cosas salen mejor y creer que puedes, es saber que sí puedes”).

En funciones de la salud mental se incluyeron en su mayoría términos positivos y algunos negativos. Los positivos fueron en torno al desarrollo de un estado de tranquilidad, paz y armonía (entre otros relacionados, una mujer citó: "sin paz en nuestro corazón, no podemos 
ser felices" y un hombre dijo: "con tranquilidad mental aprecias más y mejor lo que hay a tu alrededor"), además del logro de expresión de emociones como el amor ("si una persona no tiene amor para ella y para los demás, no tiene nada"), la felicidad y la plenitud ("sentirte bien mentalmente, te lleva a la plenitud"). Lo que en el caso de las mujeres, les permite lograr su libertad ("libre de temores"), seguridad ("la encuentra uno en las decisiones que se toman con firmeza"), independencia y congruencia; mientras que a los hombres les facilita el perdón ("si no perdonas, es como es una enfermedad que daña el alma y la mente"), la sabiduría y la realización de proyectos. Y por el contrario, como consecuencia de la falta de salud mental en las mujeres se genera tristeza ("al inicio de la terapia, trae uno mucho esa emoción"), enojo ("si tienes mucho coraje o enojo, te afecta a tu salud"), desequilibrio y malestar. Y en los hombres: celos, egoísmo, preocupación e inquietud ("se asocia a destruir la paz y la armonía en las relaciones humanas").

Finalmente, como atributos o calificativos de la salud metal, las mujeres citaron que es una necesidad y un éxito ("puedes tener éxito en cualquiera de las actividades que realizas"); los hombres que es importante y una ventaja en la vida ("es el motor principal del cuerpo humano, así el cerebro puede hacer las cosas bien").

\section{Dimensiones culturales de la salud mental}

La matriz Aggregate Proximity (AGPROX) permitió la realización de los análisis de conglomerados jerárquicos y la escala multidimensional, se presenta en la Tabla 2 para el caso de las mujeres y la Tabla 3 para los hombres.

Tabla 2

Aggregate Proximity Matrix (AGPROX) de las palabras asociadas por las mujeres

\begin{tabular}{|c|c|c|c|c|c|c|c|c|c|c|c|c|c|c|c|c|c|c|c|c|}
\hline & 1 & 2 & 3 & 4 & 5 & 6 & 7 & 8 & 9 & 10 & 11 & 12 & 13 & 14 & 15 & 16 & 17 & 18 & 19 & 20 \\
\hline 1 & 1 & & & & & & & & & & & & & & & & & & & \\
\hline 2 & .60 & 1 & & & & & & & & & & & & & & & & & & \\
\hline 3 & .75 & .70 & 1 & & & & & & & & & & & & & & & & & \\
\hline 4 & .75 & .60 & .85 & 1 & & & & & & & & & & & & & & & & \\
\hline 5 & .80 & .60 & .85 & .90 & 1 & & & & & & & & & & & & & & & \\
\hline 6 & .30 & .15 & .15 & .20 & .15 & 1 & & & & & & & & & & & & & & \\
\hline 7 & .60 & .60 & .55 & .45 & .50 & .20 & 1 & & & & & & & & & & & & & \\
\hline 8 & .25 & .10 & .10 & .15 & .10 & .85 & .15 & 1 & & & & & & & & & & & & \\
\hline 9 & .25 & .45 & .25 & .25 & .20 & .25 & .30 & .35 & 1 & & & & & & & & & & & \\
\hline 10 & .10 & .05 & .10 & .10 & .05 & .50 & .10 & .60 & .40 & 1 & & & & & & & & & & \\
\hline 11 & .15 & .15 & .20 & .25 & .25 & .15 & .25 & .15 & .15 & .35 & 1 & & & & & & & & & \\
\hline 12 & .30 & .25 & .15 & .20 & .15 & .75 & .20 & .65 & .25 & .45 & .15 & 1 & & & & & & & & \\
\hline 13 & .30 & .40 & .45 & .35 & .40 & .20 & .60 & .10 & .10 & .10 & .60 & .20 & 1 & & & & & & & \\
\hline 14 & .35 & .55 & .35 & .30 & .30 & .25 & .35 & .30 & .85 & .30 & .20 & .25 & .10 & 1 & & & & & & \\
\hline 15 & .30 & .40 & .45 & .45 & .45 & .40 & .35 & .30 & .35 & .30 & .30 & .30 & .55 & .35 & 1 & & & & & \\
\hline 16 & .35 & .30 & .45 & .30 & .35 & .30 & .65 & .20 & .20 & .20 & .40 & .20 & .75 & .20 & .55 & 1 & & & & \\
\hline 17 & .30 & .25 & .30 & .15 & .20 & .50 & .60 & .40 & .25 & .40 & .30 & .40 & .65 & .25 & .50 & .80 & 1 & & & \\
\hline 18 & .50 & .30 & .50 & .40 & .45 & .25 & .60 & .20 & .10 & .15 & .40 & .20 & .55 & .15 & .30 & .70 & .50 & 1 & & \\
\hline 19 & .55 & .35 & .55 & .65 & .60 & .30 & .30 & .20 & .05 & .05 & .40 & .30 & .35 & .15 & .30 & .35 & .20 & .40 & 1 & \\
\hline 20 & .55 & .45 & .50 & .35 & .40 & .35 & .55 & .35 & .30 & .25 & .25 & .40 & .45 & .25 & .35 & .60 & .50 & .60 & .40 & 1 \\
\hline
\end{tabular}

Nota. $1=$ Bienestar, $2=$ Felicidad, $3=$ Tranquilidad, $4=$ Paz, $5=$ Armonía, $6=$ Terapia, $7=$ Amor, $8=$ Psicólogo, $9=$ Enojo, $10=$ Loco, $11=$ Sociedad, 12=Mente, 13=Familia, 14=Tristeza, 15=Necesidad, 16=Seguridad, 17=Apoyo, 18=Paciencia, 19=Libertad, $20=$ Comprensión 
Tabla 3

Aggregate Proximity Matrix (AGPROX) de las palabras asociadas por los hombres

\begin{tabular}{|c|c|c|c|c|c|c|c|c|c|c|c|c|c|c|c|c|c|c|c|c|}
\hline & 1 & 2 & 3 & 4 & 5 & 6 & 7 & 8 & 9 & 10 & 11 & 12 & 13 & 14 & 15 & 16 & 17 & 18 & 19 & 20 \\
\hline 1 & 1 & & & & & & & & & & & & & & & & & & & \\
\hline 2 & .53 & 1 & & & & & & & & & & & & & & & & & & \\
\hline 3 & .68 & .63 & 1 & & & & & & & & & & & & & & & & & \\
\hline 4 & .47 & .58 & .68 & 1 & & & & & & & & & & & & & & & & \\
\hline 5 & .47 & .42 & .47 & .58 & 1 & & & & & & & & & & & & & & & \\
\hline 6 & .89 & .42 & .63 & .53 & .53 & 1 & & & & & & & & & & & & & & \\
\hline 7 & .37 & .32 & .37 & .53 & .68 & .53 & 1 & & & & & & & & & & & & & \\
\hline 8 & .63 & .63 & .63 & .53 & .37 & .63 & .42 & 1 & & & & & & & & & & & & \\
\hline 9 & .58 & .37 & .37 & .42 & .53 & .58 & .58 & .53 & 1 & & & & & & & & & & & \\
\hline 10 & .58 & .58 & .47 & .32 & .53 & .53 & .58 & .63 & .68 & 1 & & & & & & & & & & \\
\hline 11 & .68 & .37 & .37 & .47 & .63 & .79 & .58 & .53 & .68 & .47 & 1 & & & & & & & & & \\
\hline 12 & .63 & .42 & .74 & .63 & .68 & .68 & .42 & .47 & .53 & .42 & .63 & 1 & & & & & & & & \\
\hline 13 & .53 & .42 & .53 & .32 & .58 & .58 & .53 & .37 & .53 & .63 & .63 & .58 & 1 & & & & & & & \\
\hline 14 & .58 & .63 & .58 & .47 & .53 & .58 & .42 & .79 & .47 & .58 & .47 & .37 & .42 & 1 & & & & & & \\
\hline 15 & .42 & .53 & .53 & .53 & .68 & .37 & .42 & .47 & .53 & .63 & .42 & .58 & .68 & .53 & 1 & & & & & \\
\hline 16 & .42 & .68 & .53 & .42 & .53 & .32 & .32 & .63 & .47 & .58 & .37 & .42 & .42 & .79 & .63 & 1 & & & & \\
\hline 17 & .63 & .32 & .53 & .32 & .58 & .68 & .42 & .47 & .63 & .63 & .53 & .68 & .79 & .47 & .68 & .42 & 1 & & & \\
\hline 18 & .21 & .58 & .42 & .53 & .53 & .32 & .53 & .42 & .37 & .26 & .47 & .42 & .53 & .58 & .53 & .68 & .32 & 1 & & \\
\hline 19 & .16 & .53 & .37 & .47 & .47 & .26 & .47 & .47 & .32 & .21 & .32 & .37 & .37 & .58 & .47 & .63 & .37 & .84 & 1 & \\
\hline 20 & .47 & .58 & .58 & .53 & .42 & .53 & .37 & .42 & .47 & .47 & .47 & .63 & .74 & .32 & .63 & .37 & .74 & .47 & .42 & 1 \\
\hline
\end{tabular}

Nota. $1=$ Felicidad, 2=Conciencia, $3=$ Estabilidad, 4=Bienestar, 5=Relajación, 6=Amor, 7=Paz, 8=Perdón, 9=Entusiasmo, $10=$ Ser positivo, 11=Alegría, 12=Tranquilidad, 13=Sabiduría, 14=Paciencia, 15=Equilibrio, 16=Conciencia, $17=$ Plenitud, 18=Psicólogo, 19=Psiquiatra, $20=$ Salud

El dendograma con la agrupación de las palabras que realizaron las mujeres se muestra en la Figura 1. Para las participantes el concepto de salud mental se compone de dos grandes dimensiones: "necesidades" y "experiencias". La primera se subdividió en "expresión de sentimientos" (incluyó estabilidad y valores) y "proyecto de vida" la cual incluyó "apoyo social" (seguridad y habilidades sociales) y "la sociedad te limita". La dimensión "experiencias" se subdividió en “apoyo psicológico” y “depresión” (incluyó: no debería existir el enojo, ni la tristeza).

El dendograma generado de la agrupación que realizaron los hombres se muestra en la Figura 2. El concepto de salud mental se compone de dos grandes dimensiones: "armonía" y "bienestar". La primera se subdividió a su vez en "salud emocional" (incluyó vida), "equilibrio" (comprende: salud física y optimismo) y "autoconocimiento" (felicidad). La dimensión de "bienestar" se subdividió en "futuro" (incluyó: tranquilidad, inicio de salud mental y terapia) y "tener trabajo".

\section{Consenso cultural del concepto de salud mental}

El archivo Individual proximities (INDPROX) permitió la realización de los análisis de consenso, se presenta en la Tabla 4 para ambos grupos de participantes.

La organización conceptual de las dimensiones del concepto de salud mental se presenta en la Tabla 5. En el caso de las mujeres alcanzó un nivel de razón 5.54 y en los hombres 1.45. Como ya se mencionó, la regla es que este factor (verdad común) debe calificar más de tres veces la 


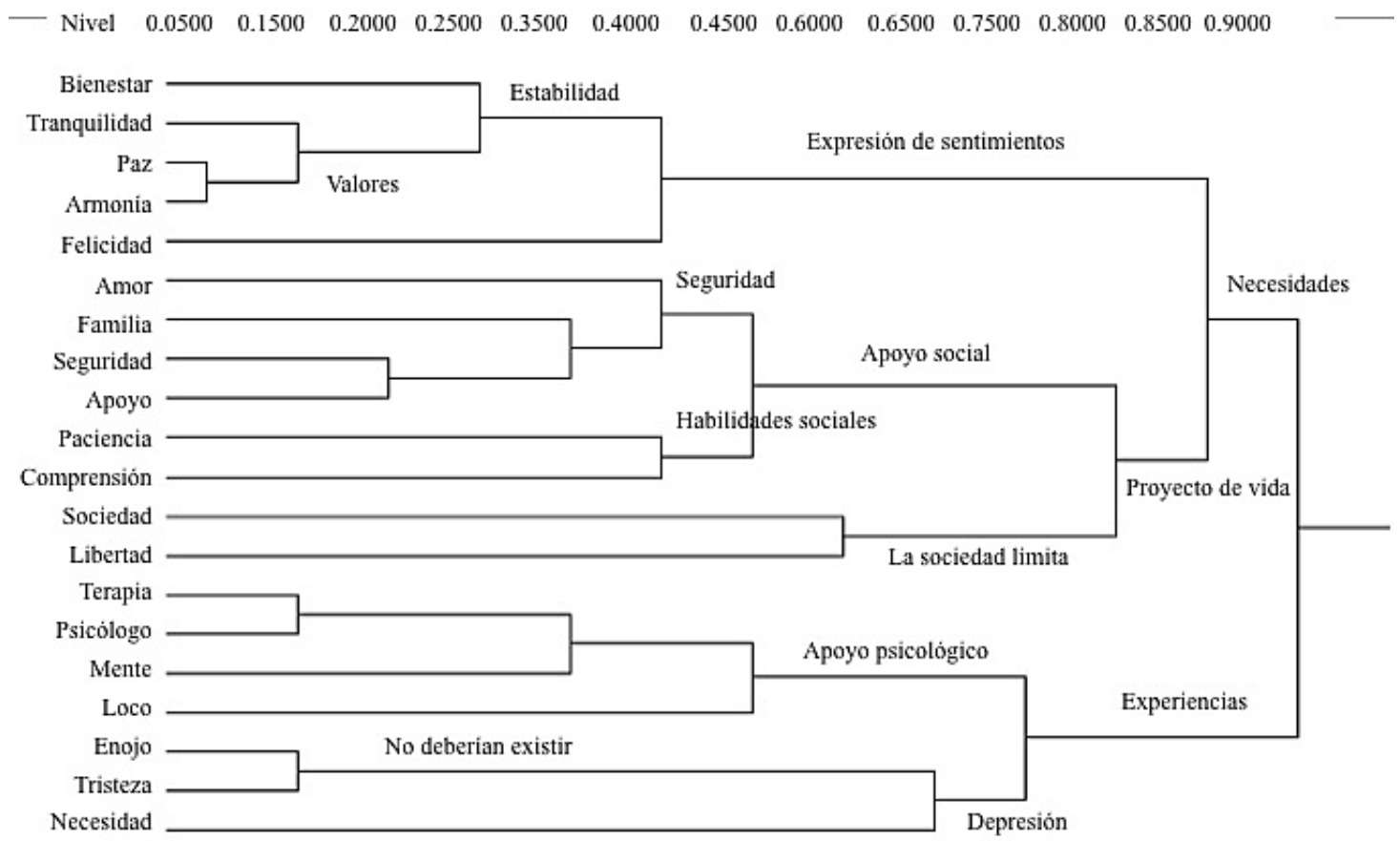

Figura 1. Dimensiones culturales del concepto de salud mental de mujeres, Guadalajara, México, 2013. El nivel indica el grado de asociación (similaridad) entre ítems dentro de un cluster. Por ejemplo, el nivel 0.2500 indica que el promedio de distancia entre los ítems dentro del cluster es de 0.2500 .

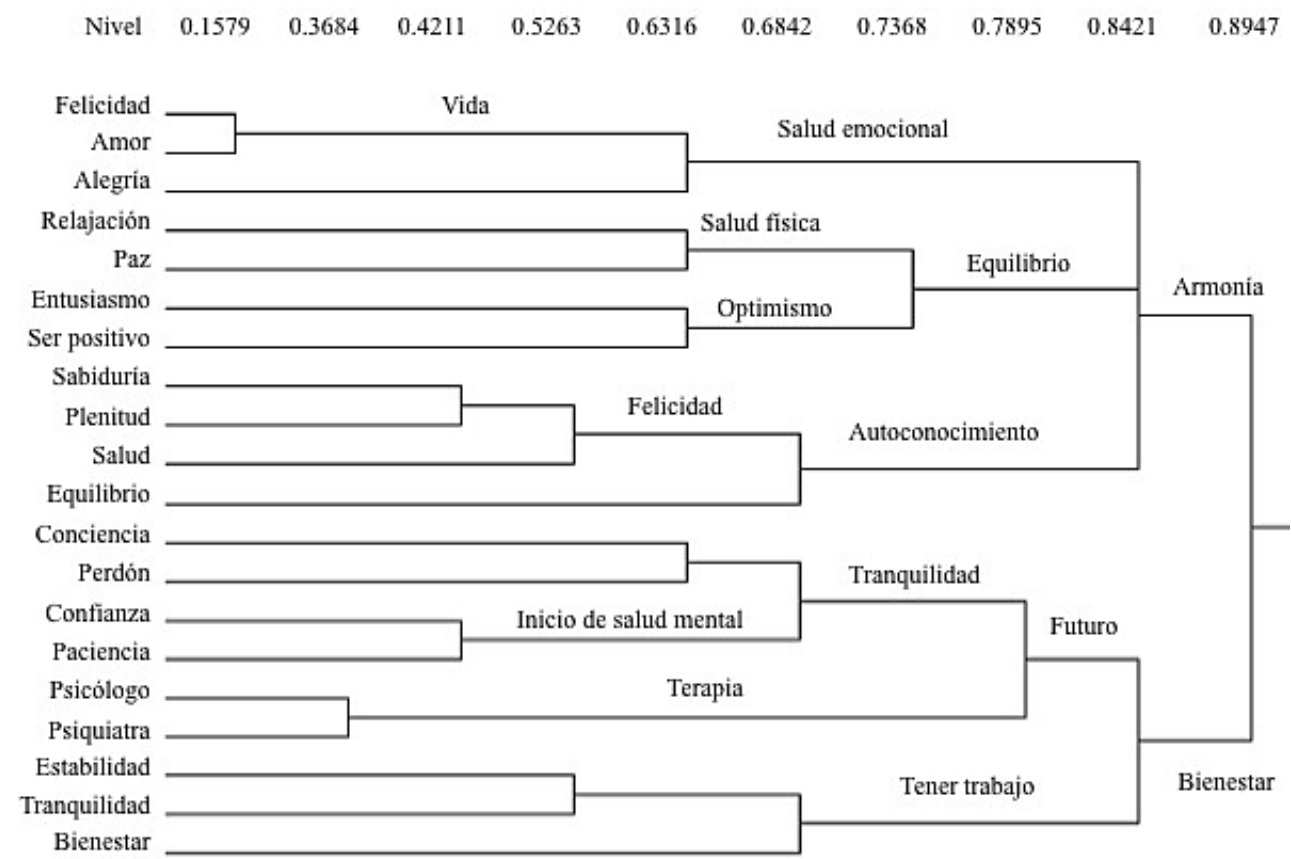

Figura 2. Dimensiones culturales del concepto de salud mental de hombres, Guadalajara, México, 2013. El nivel indica el grado de asociación (similaridad) entre ítems dentro de un cluster. Por ejemplo, el nivel 0.6316 indica que el promedio de distancia entre los ítems dentro del cluster es de 0.6316. 
Tabla 4

Individual aggregate matrix (INDPROX). Correlaciones entre cada participante y la aggregate matrix

\begin{tabular}{ccc}
\hline Participantes & Mujeres & Hombres \\
\hline 1 & .310 & .246 \\
2 & .414 & .259 \\
3 & .375 & .199 \\
4 & .347 & .365 \\
5 & .235 & .227 \\
6 & .639 & .351 \\
7 & .425 & .369 \\
8 & .362 & .324 \\
9 & .549 & .238 \\
10 & .285 & .225 \\
11 & .269 & .243 \\
12 & .488 & .110 \\
13 & .551 & .233 \\
14 & .370 & .252 \\
15 & .335 & .355 \\
16 & .544 & .177 \\
17 & .450 & .438 \\
18 & .514 & .200 \\
19 & .572 & .211 \\
20 & .473 & \\
\hline
\end{tabular}

varianza del segundo factor (homogenidad del ítem), lo cual sucedió sólo en el caso de las mujeres (3.61 veces mayor). El porcentaje acumulado de la varianza para el factor ratio 1 fue mayor a 70\% (88.2 y 81.7) lo que también valida el consenso. Y las dimensiones descritas en los árboles de conglomerados tuvieron valores de bondad de ajuste adecuados (stress .135 mujeres y .200 hombres) en el análisis de escala multidimensional no métrica.

\section{Discusión}

Se logró el objetivo de analizar las concepciones culturales sobre la salud mental de personas adultas que asisten a procesos terapéuticos psicológicos de Guadalajara, México.

Para las personas participantes, la salud mental es el bienestar, la salud y la cordura que se logra con el apoyo de profesionales y de instituciones de salud. Es un estado de equilibrio, tranquilidad, paz y armonía, además del logro de expresión de emociones como el amor, la felicidad y la plenitud. Ello les permite obtener felicidad, entusiasmo, seguridad y congruencia. Por el contrario, la falta de salud mental puede ocasionar algunos trastornos mentales (neurosis, psicosis, depresión).

Esta conceptualización coincide con el estudio de Mora et al. (2003) que destacó la importancia de la

Tabla 5

Niveles de consenso cultural sobre el concepto de salud mental

\begin{tabular}{|c|c|c|c|c|c|c|c|c|}
\hline & \multirow{2}{*}{$\begin{array}{c}\text { Razón del } \\
\text { Factor } \\
1(\mathrm{~F} 1) \\
\end{array}$} & \multirow{2}{*}{$\begin{array}{c}\text { Varianza } \\
\text { acumulada } \\
\% \\
\end{array}$} & \multirow{2}{*}{$\begin{array}{c}\text { Razón del } \\
\text { Factor } \\
2(\mathrm{~F} 2) \\
\end{array}$} & \multirow{2}{*}{$\begin{array}{c}\text { Varianza } \\
\text { acumulada } \\
\% \\
\end{array}$} & \multirow{2}{*}{$\begin{array}{l}\text { Razón* } \\
\text { F1:F2 }\end{array}$} & \multicolumn{2}{|c|}{ Competencia cultural** } & \multirow{2}{*}{$\begin{array}{l}\text { Concordancia } \\
\text { grupal }^{* * *}\end{array}$} \\
\hline & & & & & & Media & $\mathrm{DE}$ & \\
\hline $\begin{array}{l}\text { Mujeres } \\
(\mathrm{n}=20)\end{array}$ & 5.54 & 69.0 & 1.54 & 88.2 & 3.6 & .471 & .236 & .221 \\
\hline $\begin{array}{c}\text { Hombres } \\
(\mathrm{n}=19)\end{array}$ & 1.45 & 42.4 & 1.347 & 81.7 & 1.0 & .236 & .144 & .056 \\
\hline
\end{tabular}

Nota. ${ }^{*}$ Cociente de F1 en relación con F2. La regla es que F1 sea por lo menos tres veces mayor que F2 para hacer válido el consenso. ${ }^{*}$ Media aritmética grupal del grado de acuerdo individual con las respuestas promedio. Concordancia entre las respuestas individuales y el patrón estimado de respuestas correctas del grupo. ***Acuerdo grupal en las respuestas obtenidas por los informantes. Se obtiene elevando al cuadrado el promedio de la competencia cultural (Weller, 2007).

Actualidades en Psicología, 29(118), 2015, 35-46 
tranquilidad, la confianza, el cariño y la compresión como elementos base de la salud mental; y con el hallazgo de Åsbring (2012) sobre la importancia del equilibrio en la vida diaria como generador de buena salud mental. Además, la visión holística de la salud mental coincide también con lo encontrado por otros estudios sobre la conceptualización de la salud en general (Torres-López, Munguía, Pozos \& Aguilera, 2010).

Se observaron además, diferencias de género en las concepciones culturales de la salud mental de los participantes. Las que son importantes en la implementación de programas de promoción para la salud mental, ya que el uso de la perspectiva género permitiría analizar las formas simbólicas de que se vale la cultura para institucionalizar las diferencias entre hombres y mujeres (Lamas, 2001).

En el caso de las mujeres se destacó el haber mostrado un mayor consenso en las dimensiones conceptuales de la salud mental. Ello indica una construcción más sólida del mismo, lo que puede tener como base el papel social de la mujer como uno de los principales actores en la búsqueda y atención de la salud en general ( $y$ en este caso de la salud mental).

$\mathrm{El}$ incluir aspectos sociales o comunitarios como son la familia, la comunidad y la escuela, así como la búsqueda de orientación psicológica a fin de lograr su libertad, seguridad e independencia, son algunos de los elementos distintivos de género. Estos aspectos fueron señalados por Berenzón \& Mora (2005) en particular en la asociación de la libertad a la salud mental.

Además, en las dimensiones de la salud mental, las mujeres destacaron la necesidad de expresión de sus emociones (ya que de no suceder esto se puede generar tristeza, enojo, desequilibrio y malestar) y del apoyo social a fin de obtener seguridad y otras habilidades sociales. Otra base son sus experiencias al recibir orientación psicológica, lo que les permite un mejor manejo de sus emociones, calificadas como negativas. Es decir, que desde su perspectiva el proceso terapéutico constituye una forma de desarrollar habilidades de comunicación de emociones que de otra forma estarían reprimidas.

Los hombres mostraron un menor consenso en su concepción cultural de la salud mental. Indica que para este grupo genérico, el dominio cultural del concepto se encuentra en proceso de construcción, a pesar de ya haber buscado y recibido atención psicológica. No obstante, resaltaron la importancia que tiene en la armonía y paz obtenidas por la mejora de su salud emocional y el autoconocimiento. Además, el bienestar generado les proporciona cierta seguridad para su futuro, con la tranquilidad de estar en camino de obtener su salud mental (con el apoyo de la terapia psicológica).

En contraposición, para ellos la falta de salud mental les genera celos, egoísmo, preocupación e inquietud. Como elemento distintivo incluyeron la importancia del desarrollo de ejercicio físico, el ser positivo y la realización de proyectos (esto último también encontrado en el estudio de Åsbring, 2012).

Llama la atención que ninguno de los participantes hizo referencia a algún componente de tipo espiritual asociado a la conceptualización de la salud mental, ello a pesar de haber sido reportado en estudios previos en población mexicana (Berenzón \& Mora, 2005; Torres-López et al., 2010).

En suma, en las dimensiones culturales de la salud mental de los participantes del estudio se encontraron componentes relacionados con su experiencia de participación en procesos terapéuticos psicológicos. $\mathrm{Al}$ parecer, constituye una importante estrategia de promoción para la salud mental disponible para este tipo de población, en un nivel individual.

Convendría desarrollar más estrategias en los niveles grupal, institucional y social como parte de una promoción para la salud mental más efectiva. Así, uno de los retos es promover una visión más comunitaria de la salud mental, con el fin de generar conciencia que en esta área no sólo es el resultado de acciones individuales, sino por todo lo que rodea al individuo, es decir su ambiente familiar, laboral y social. Con ello se tendría un impacto más exitoso de lo que se ha logrado hasta ahora.

Otra de las aportaciones del estudio es la metodología empleada, ya que presenta la ventaja de utilizar técnicas de aplicación y análisis de resultados en forma más rápida que las utilizadas en los estudios cualitativos tradicionales. Por lo cual, la transferibilidad metodológica (Flick, 2014) podría resultar de interés en otros campos disciplinares. 
Las limitaciones de este estudio son el énfasis en los elementos cognitivos mientras que los procesos participativos y prácticas no son considerados. Para futuros estudios se siguiere incluir otros tipos de población, estratificando por grupos de edad y diferentes condiciones sociales.

\section{Referencias}

Åsbring, P. (2012). Words about body and soul: Social representations relating to health and illness. Journal of Health Psycholgy, 17, 1110-1120.

Bernard, H. R. (2006). Research Methods in cultural Anthropology. Newbury Park: Sage Publications.

Berenzón, S., \& Mora, J. (2005). Los padeceres emocionales cotidianos: percepciones y creencias en dos poblaciones de la ciudad de México. Investigación en salud, 7(3), 181-187.

Flick, U. (2014). La gestión de la calidad en investigación cualitativa. Madrid: Ediciones Morata.

Lamas, M. (2001). Cuerpo: diferencia sexualy génera. México: Taurus.

Marseline, K. S. J., \& Premalatha, A. (2013). An NMF and Hierarchical Based Clustering Approach to support Multi view point-Based Similarity Measure. International Journal of Computer Trends and Technology, 41(3), 285-291.

Mora, J., Flores, F., De Alba, M., \& Marroquín, M. (2003). Construcción de significados acerca de la salud mental en población adulta de una comunidad urbana marginal. Salud Mental, 26(5), 51-60.

Organización Mundial de la Salud (OMS). (2004). Promoting mental health: concepts, emerging evidence, practice. Recuperado de http://www.who.int/mental_health/ evidence/en/promoting_mhh.pdf.

Organización Mundial de la Salud (OMS). (2005). Promoción de la salud mental: conceptos, evidencia emergente, práctica. Recuperado de http://www.who.int/mental_health/ evidence/promocion_de_la_salud_mental.pdf

Organización Mundial de la Salud (OMS). (2013). Plan de acción sobre salud mental 2013-2020. Recuperado de www.who.int/ iris/bitstream/10665/97488/1/9789243506029_spa.pdf.
Ruiz, J.I., Ponce de León, E., \& Herrera, A.N. (2001). Avances en Medición Evaluación en Psicología y Educación. Bogotá: Universidad del Bosque.

Sturrock, K., \& Rocha, J.A. (2000). Multidimensional Scaling Stress evaluation table. Field Methods, 12, 49-60.

Suri, H. (2011). Purposeful Sampling in Qualitative Research Synthesis. Qualitative Research Journal, 11(2), 63-75.

Torres-López, T.M., Munguía, J., Pozos, B.E., \& Aguilera, M.A. (2010). Representaciones sociales sobre la salud y la enfermedad de la población adulta de Guadalajara, México. Atención Primaria, 42(3),154-161.

Torres-López, T.M., Guerrero, J., \& Salazar, J.G. (2012). Dimensiones conceptuales del dengue que favoreces o limitan su prevención. Revista Panamericana de Salud Pública, 31(3), 197-203.

Torres-López, T.M., Matsui, J.O., \& Aranda, C. (2012). Dimensiones culturales del concepto de influenza humana en estudiantes y docentes de ciencias de la salud que favorecen o dificultan su prevención. Desacatos, 39, 45-56.

Torres-López, T.M., Soltero, R., \& Herrera, J.I. (2012). Dimensiones culturales sobre el dengue en profesionales de la salud de México y Colombia. Rev. Salud Pública, 14(1), 67-80.

Torres-López, T.M., \& Munguía, J.A. (2012). Concepciones culturales del VIH/Sida de jóvenes que son parte de grupos culturales juveniles de Guadalajara, México. Revista CES Psicología, 5(2), 119-133.

Weller, S.C., \& Mann, N.C. (1997). Assesing rater performance without a gold standard using consensus theory. Med Decis Making, 17(1), 71-9.

Weller, S.C. (2007). Questions Cultural Consensus Theory: Applications and Frequently Asked. Field Methods, 19, 339-368. 